\title{
CONSUMO DE COMBUSTÍVEL NA OPERAÇÃO DE SUBSOLAGEM REALIZADA ANTES E DEPOIS DE DIFERENTES SISTEMAS DE PREPARO PERIÓDICO DO SOLO
}

\author{
NILSON SALVADOR ${ }^{1}$, SÉRGIO H. BENEZ ${ }^{2}$, RENILDO L. MION ${ }^{3}$
}

RESUMO: A subsolagem tem aumentado nos últimos anos de forma indiscriminada, faltando estudos que norteiem os melhores procedimentos para que novos problemas não sejam acrescentados devido a subsolagens inadequadas ou mesmo em solos onde a operação é desnecessária, e principalmente buscar redução no consumo de combustível. Dessa forma, este trabalho teve o objetivo de avaliar o consumo de combustível na operação de subsolagem efetuada antes e depois de diferentes sistemas de preparo periódico do solo, classificado como Nitossolo Vermelho distroférrico. Os preparos foram realizados com arado de discos, arado de discos mais uma gradagem de nivelamento, grade pesada, grade pesada mais gradagem de nivelamento e escarificador. A realização da subsolagem depois dos sistemas de preparo periódico requereu 15\% menos de potência na barra de tração. A subsolagem depois dos diferentes sistemas de preparo economizou $16,5 \%$ de combustível por área. O deslizamento das rodas motrizes e a velocidade média operacional obtiveram melhor desempenho quando se realizou a subsolagem depois do preparo do solo.

PALAVRAS-CHAVE: subsolador, potência, implementos, deslizamento das rodas motrizes, velocidade operacional.

\section{FUEL CONSUMPTION IN THE SUBSOILING OPERATION ACCOMPLISHED BEFORE AND AFTER DIFFERENT PREPARATION SYSTEMS OF PERIODIC SOIL TILLAGE}

\begin{abstract}
The subsoiling has had a great increase in the last few years, but there is still a lack of studies that guide the best procedures so that new problems do not appear because of inadequate subsoiling or even in soil where the same operation is unnecessary, and primarily look for a reduction in the consumption of fuel. Thus, this work had the objective of evaluating the fuel consumption in the operation of subsoiling performed before and after different systems of preparation of a regular land classified as Nitossolo Red Distroférrico. The periodic tillage systems were: plowing with disks, plowing with disks followed by one leveling, plowing harrow, plowing harrow followed by leveling and chiseling. The accomplishment of the subsoiling after the preparation of periodic systems required $15 \%$ less power in the bar of traction. The subsoiling after the different systems of preparation saved $16.5 \%$ of fuel per area. The slip of the driving wheels and average operational speed obtained better performance when it took the subsoiling after preparing the soil.
\end{abstract}

KEYWORDS: subsoiling, power, implements, slip of the driving wheels, operational speed.

\section{INTRODUÇÃO}

A utilização da operação de subsolagem tem aumentado nos últimos anos de forma indiscriminada, faltando estudos que norteiem os melhores procedimentos para que novos problemas não sejam acrescentados devido a subsolagens inadequadas ou mesmo em solos onde a operação é desnecessária, e principalmente buscar redução no consumo de energia.

\footnotetext{
${ }^{1}$ Prof. Adjunto de Máquinas e Mecanização Agrícola, DEG/UFLA, Lavras - MG.

${ }^{2}$ Prof. Titular de Máquinas e Mecanização Agrícola, FCA/UNESP, Botucatu - SP.

${ }^{3}$ Prof. Adjunto, Departamento de Engenharia Agrícola, DENA/UFC, Fortaleza - CE, rmion@ufc.br

Recebido pelo Conselho Editorial em: 6-3-2007

Aprovado pelo Conselho Editorial em: 27-2-2008
} 
A subsolagem é uma operação de preparo do solo que serve para romper e/ou quebrar camadas compactadas formadas nas camadas inferiores do solo, causadas pelo intenso cultivo das culturas, utilizando cada vez máquinas mais pesadas e maiores, de forma a diminuir o tempo gasto nas operações agrícolas.

Dessa forma, fica evidente que está ocorrendo acentuada degradação, compactação dos solos e uso indiscriminado da subsolagem. Segundo EL-ARABY et al. (1987), a subsolagem não deve ser caracterizada como preparo periódico do solo, pois deve-se utilizar quando se verifica a existência de camadas compactadas no perfil do solo.

LANÇAS (1987), ao estudar os efeitos da velocidade de avanço na operação de subsolagem, concluiu que o aumento da velocidade proporcionou maiores rendimentos operacionais com menor consumo de combustível, porém a força de tração foi maior para velocidades operacionais maiores, independentemente do tipo de haste e de ponteira. LANÇAS (1988) também avaliou o desempenho de três formas geométricas de hastes: reta inclinada a $45^{\circ}$ e duas formas parabólicas; dois tipos de ponteira: com e sem asas, e três montagens com diferentes números de hastes: $1 ; 2$ e 3, concluindo, em função da média dos blocos, que a haste parabólica marca Maschietto, na montagem com três hastes equipadas com ponteiras aladas, apresentou melhores resultados de consumo específico operacional e resistência específica operacional.

A demanda de potência na operação de subsolagem sofre influência de inúmeras variáveis. FORNSTRON \& BECKER (1977) verificaram em seus estudos grande variação na demanda de maquinaria para os mesmos implementos em um mesmo solo. Segundo esses autores, a tração requerida pela maquinaria é muito variável com o estado do solo e particularmente com o teor de água do mesmo. A eficiência na unidade de potência foi altamente correlacionada à potência requerida na barra.

Para LYNE et al. (1984), a otimização do desempenho de um trator requer um sistema de controle para a seleção apropriada dos valores relacionados ao motor e aos seus parâmetros de tração. A previsão precisa de energia ou da força requerida pelas ferramentas de preparo tem sido prejudicada devido às dificuldades na identificação de parâmetros relevantes no sistema soloferramenta, principalmente os parâmetros de resistência dinâmica do solo.

Estudando pneus novos e usados com rotações no motor de $1.800 \mathrm{rpm}$ e $2.000 \mathrm{rpm}$, MIRANDA et al. (2000) observaram que pneus desgastados e rotação no motor de $2.000 \mathrm{rpm}$ proporcionaram maior velocidade de deslocamento e tempo operacional teórico $6,8 \%$ menor; entretanto, a rotação de $1.800 \mathrm{rpm}$ foi mais vantajosa economicamente, pois, além de reduzir a patinagem em 19,2\%, obteve-se consumo horário de combustível 12,9\% menor e, por hectare, $2,32 \mathrm{~L}$ menor, ou 7\%. Com o uso de pneus novos, todos os parâmetros tiveram melhor comportamento, mas a tendência foi semelhante e, quando se trabalhou a $1.800 \mathrm{rpm}$, o que mais se evidenciou, foi a redução de $26,9 \%$ na patinagem e de $10,5 \%$ no consumo horário de combustível, mas a diferença de 1,56 L a menos no consumo de combustível por hectare não foi significativa.

Estudando o desempenho energético de subsoladores, KICHLER et al. (2007) observaram que ocorreu aumento no consumo de combustível de $20 \%$ e incremento na força de $120 \%$ quando se aumentou a profundidade de $23 \mathrm{~cm}$ para $35 \mathrm{~cm}$.

RAPER et al. (2005) verificaram redução no consumo de combustível da ordem de $43 \%$ e $27 \%$, quando a profundidade de subsolagem foi de 25 e $35 \mathrm{~cm}$, respectivamente, comparado à profundidade de $45 \mathrm{~cm}$. O mesmo autor alerta que se pode conseguir redução do consumo de combustível trabalhando com profundidades variáveis, de acordo com a camada do solo a ser descompactada.

O objetivo deste trabalho foi estudar o consumo de combustível da subsolagem realizada antes e depois de diferentes sistemas de preparo periódico do solo. 


\section{MATERIAL E MÉTODOS}

O ensaio foi instalado e conduzido na Fazenda Experimental Lageado, Faculdade de Ciências Agronômicas, UNESP, Câmpus de Botucatu, Estado de São Paulo. A área experimental foi definida pelas seguintes coordenadas geográficas: $22^{\circ} 49^{\prime} 31^{\prime \prime}$ sul e $48^{\circ} 25^{\prime} 37^{\prime \prime}$ oeste. A altitude e a declividade média são de $770 \mathrm{~m}$ e $2,5 \%$, respectivamente. Essa área vinha sendo cultivada há vários anos com a cultura do milho, utilizando-se ininterruptamente do sistema convencional de preparo do solo (aração com arado de disco e gradagem niveladora leve).

A área útil de cada parcela foi de $150 \mathrm{~m}^{2}$, isto é, $30 \mathrm{~m}$ de comprimento por $5 \mathrm{~m}$ de largura, sendo os tratamentos delineados em blocos casualizados, em esquema fatorial ( 5 x 2), com cinco repetições, com os seguintes métodos de preparo periódico: uma aração com arado de discos (D); aração com arado de discos mais uma gradagem niveladora (Dn); gradagem pesada (G); uma gradagem pesada mais uma gradagem niveladora $(\mathrm{Gn})$, e escarificação $(\mathrm{E})$, em duas condições de subsolagem: antes (AP) e depois (DP) do preparo periódico do solo, respectivamente, com cinco repetições.

O solo utilizado para as determinações foi classificado como Nitossolo Vermelho distroférrico, textura argilosa (EMBRAPA, 1999). As operações de preparo periódico foram realizadas 15 dias antes da subsolagem, quando o solo apresentava, em média, teor de água de 19,8 e $26,2 \%$ nas camadas de 0 a 10 e de 11 a $20 \mathrm{~cm}$ de profundidade, e a densidade do solo foi de 1,40 e $1,41 \mathrm{~g} \mathrm{~cm}^{-3}$ nas diferentes camadas, respectivamente. Independentemente das condições de subsolagem, manteve-se a mesma marcha no trator, rotação do motor e regulagens no subsolador. A profundidade média de trabalho do arado, grade pesada e escarificador, tomados de $50 \mathrm{em} 50 \mathrm{~mm}$ no sentido transversal ao da faixa trabalhada, foi: 185; 160 e $169 \mathrm{~mm}$, respectivamente, enquanto a do subsolador foi de $312 \mathrm{~mm}$.

As características dos equipamentos utilizados são as seguintes: a) arado de arrasto, marca SANS, modelo $1 \mathrm{M}$, com quatro discos de $711 \mathrm{~mm}$ de diâmetro e peso de $1.350 \mathrm{kgf}$; b) grade pesada - tipo "off set", com dez discos de $812,8 \mathrm{~mm}$ e peso de $3.508 \mathrm{kgf}$; c) grade niveladora - tipo "off set", com 32 discos de $508 \mathrm{~mm}$ de diâmetro, espaçadas entre si de $167 \mathrm{~mm}$, peso de $687 \mathrm{kgf}$; d) escarificador - equipado com dez hastes, distribuídas em três barras-ferramentas, ponteiras de 50 mm de largura e ângulo de ataque de $20^{\circ}$, com peso de $446 \mathrm{kgf}$; e) subsolador de arrasto dotado de três hastes parabólicas com ponteiras aladas, espaçadas de $700 \mathrm{~mm}$, dispostas triangularmente, com peso de $710 \mathrm{kgf}$; f) trator Valmet 128 (4 x 2 TDA), com potência na TDP à rotação nominal no motor de 76,5 kW, lastrado, peso de $6.900 \mathrm{kgf}$, pneus dianteiros R-1 (14.9x26) - pressão de inflação de $95 \mathrm{kPa}$ - e traseiros R-1 (18.4x34) - pressão de inflação de $110 \mathrm{kPa}$. Durante todas as operações de campo, manteve-se a rotação do motor do trator em $1.750 \mathrm{rpm}$.

Foram utilizados os seguintes equipamento para a medição da força de tração: a) célula de carga, modelo U1, precisão $\pm 0,3 \%$, capacidade de $5 \mathrm{tf}$, fabricada pela HBM, instalada entre a barra de tração e o cabeçalho de cada implemento; b) indicador de força instantânea, modelo MVD/2405, capacidade de quatro dígitos, precisão \pm 1 dígito, fabricado pela HBM. Os aparelhos, bem como um assento para o instrumentador, foram posicionados na parte posterior do trator; c) indicador de força integrada composto de conversor de voltagem/freqüência, confeccionado no Centro Nacional de Engenharia Agrícola - CENEA, Sorocaba, São Paulo; d) freqüencímetro marca New Port, modelo 6220 , e cronômetro modelo EAA-110, com base quartzo e precisão $\pm 0,01$ segundo, fabricado pela Eletronic Assembly.

O consumo de combustível na subsolagem foi obtido com aparelho de medição direta, denominado Pierburg, modelo PLU 106, com capacidade de registro de até $60 \mathrm{~L} \mathrm{~h}^{-1}$, com indicador analógico e contador eletromecânico que fornece o volume de combustível consumido por segundo. A massa específica do óleo diesel utilizado nos ensaios foi de $887 \mathrm{~g} \mathrm{~L}^{-1}$ à temperatura de $20{ }^{\circ} \mathrm{C}$ e viscosidade média de 5,53 centipoise a $23,2{ }^{\circ} \mathrm{C}$. 
A força média de tração na subsolagem foi obtida utilizando a força integrada e o tempo gasto para percorrer $30 \mathrm{~m}$, que também foi utilizado para a obtenção da velocidade de deslocamento, permitindo, ainda, o cálculo da potência na barra de tração.

O consumo horário de combustível foi calculado por meio das leituras obtidas pelo Pierburg, que forneceu o volume em $\mathrm{cm}^{3}$, consumido no percurso de $30 \mathrm{~m}$. Esse volume foi convertido em $\mathrm{L} \mathrm{ha}^{-1}$, e no consumo específico, em $\mathrm{g} \mathrm{kWh}{ }^{-1}$. O consumo total de combustível por hectare, para as seqüências operacionais, foi obtido somando-se o combustível gasto por hectare em cada tratamento periódico do solo e aquele consumido na operação de subsolagem correspondente, em cada uma das seqüências operacionais.

\section{RESULTADOS E DISCUSSÃO}

Observando os resultados obtidos na Tabela 1, da potência requerida para a subsolagem depois do preparo (DP), a maior demanda de potência foi para o sistema de preparo Gn, devido à maior exigência de força de tração. Comparando os resultados da potência das condições de subsolagem, dentro de cada sistema de preparo, pode-se observar que, à exceção do sistema de preparo Gn, a subsolagem depois do preparo (DP) foi estatisticamente melhor; em média, a exigência de potência foi menor cerca de $15 \%$ em relação à condição antes do preparo (AP).

Com o melhoramento na eficiência trativa dos tratores de pneumáticos agrícolas, podem-se usar tratores menores para a operação de subsolagem depois do preparo do solo, o que, segundo BURT \& LYNE (1989), melhora a produtividade das culturas, reduz a compactação do solo e os custos operacionais diretos.

TABELA 1. Potência média na barra de tração requerida pelo subsolador, em função das condições de preparo. Average potency on the draw-bar required $(\mathrm{kW})$, by the subsoiler operation performed before and after the periodic soil tillage.

\begin{tabular}{lccc}
\hline & \multicolumn{3}{c}{ Potência na Barra de Tração (kW) } \\
\cline { 2 - 3 } Sistemas de Preparo & \multicolumn{2}{c}{ Condições de Subsolagem } & \multirow{2}{*}{ Diferença (\%) } \\
\cline { 2 - 3 } $\mathrm{D}$ & \multicolumn{1}{c}{$\mathrm{AP}$} & $\mathrm{DP}$ & $-16,2$ \\
$\mathrm{Dn}$ & $23,68 \mathrm{Ab}$ & $19,76 \mathrm{Aa}$ & $-19,2$ \\
$\mathrm{G}$ & $24,13 \mathrm{Ab}$ & $19,50 \mathrm{Aa}$ & $-15,1$ \\
$\mathrm{Gn}$ & $23,90 \mathrm{Ab}$ & $20,59 \mathrm{Aa}$ & $-2,8$ \\
$\mathrm{E}$ & $23,24 \mathrm{Aa}$ & $22,58 \mathrm{Ba}$ & $-21,1$ \\
\hline Média & $24,07 \mathrm{Ab}$ & $19,00 \mathrm{Aa}$ & $-15,0$ \\
\hline
\end{tabular}

Médias seguidas de mesma letra maiúscula na coluna e de mesma letra minúscula na linha não diferem entre si, a 5\% de probabilidade, pelo teste de Tukey.

Os resultados da Tabela 2 mostram que, dentro de cada condição de subsolagem, a quantidade de combustível necessária para subsolar um hectare foi estatisticamente a mesma para todos os sistemas de preparo. Todavia, ao confrontar o gasto de combustível numa condição e em outra, dentro de cada sistema de preparo, verifica-se que, à exceção do sistema de preparo G, a subsolagem depois do preparo (DP) foi estatisticamente superior para todos os tratamentos de preparo. De modo geral, a subsolagem depois do preparo (DP) proporcionou economia média de $16,5 \%$. Esse resultado permite concluir que, com a quantidade de combustível necessária para subsolar um hectare, usando-se o método tradicional - condição (AP) -, pode-se subsolar área $16,5 \%$ maior se utilizar o método modificado, ou seja, subsolagem depois do preparo (DP).

Observa-se, na Tabela 3, que os valores médios do consumo específico de combustível na subsolagem não sofreram influência das diferentes condições dos sistemas de preparo; todavia, verifica-se pelos resultados que a otimização simultânea do desempenho do motor e a eficiência trativa para o conjunto trator-subsolador foram melhores na condição de subsolagem antes do preparo (AP). 
TABELA 2. Volume de combustível requerido por área, em função das condições de subsolagem e dos sistemas de preparo do solo. Fuel required by area $\left(\mathrm{L} \mathrm{ha}^{-1}\right)$, depending on the conditions of subsoiling and systems of soil preparation.

\begin{tabular}{lccc}
\hline \multirow{2}{*}{ Sistemas de Preparo } & \multicolumn{2}{c}{ Consumo de Combustível $\left(\mathrm{L} \mathrm{ha}^{-1}\right)$} & \\
\cline { 2 - 3 } & \multicolumn{2}{c}{ Condições de Subsolagem } & Diferença (\%) \\
\hline $\mathrm{D}$ & $27,42 \mathrm{Ab}$ & $\mathrm{DP}$ & $-22,6$ \\
$\mathrm{Dn}$ & $27,75 \mathrm{Ab}$ & $21,21 \mathrm{Aa}$ & $-23,6$ \\
$\mathrm{G}$ & $25,81 \mathrm{Aa}$ & $24,12 \mathrm{Aa}$ & $-6,6$ \\
$\mathrm{Gn}$ & $27,23 \mathrm{Ab}$ & $22,80 \mathrm{Aa}$ & $-16,3$ \\
$\mathrm{E}$ & $26,54 \mathrm{Ab}$ & $22,90 \mathrm{Aa}$ & $-13,7$ \\
\hline Média & $26,95 \mathrm{~b}$ & $22,48 \mathrm{a}$ & $-16,5$ \\
\hline
\end{tabular}

Médias seguidas de mesma letra maiúscula na coluna e de mesma letra minúscula na linha não diferem entre si, a $5 \%$ de probabilidade, pelo teste de Tukey.

TABELA 3. Consumo específico de combustível, em função das condições de subsolagem e dos sistemas de preparo periódico do solo. Specific consumption of fuel $\left(\mathrm{g}_{\mathbf{k W ~ h}} \mathbf{h}^{-1}\right)$, depending on the conditions of subsoiling and systems of soil preparation.

\begin{tabular}{lccc}
\hline \multicolumn{3}{c}{ Consumo Específico de Combustível $\left(\mathrm{g} \mathrm{kW} \mathrm{h}^{-1}\right)$} \\
\cline { 2 - 3 } Sistemas de Preparo & \multicolumn{2}{c}{ Condições de Subsolagem } & \multirow{2}{*}{ Diferença (\%) } \\
\cline { 2 - 3 } $\mathrm{D}$ & $\mathrm{AP}$ & $\mathrm{DP}$ & 2,39 \\
$\mathrm{Dn}$ & $446,2 \mathrm{Aa}$ & $456,8 \mathrm{Aa}$ & 7,33 \\
$\mathrm{G}$ & $435,5 \mathrm{Aa}$ & $467,4 \mathrm{Aa}$ & 12,99 \\
$\mathrm{Gn}$ & $430,2 \mathrm{Aa}$ & $486,1 \mathrm{Aa}$ & $-2,35$ \\
$\mathrm{E}$ & $452,4 \mathrm{Aa}$ & $441,7 \mathrm{Aa}$ & 15,66 \\
\hline Média & $430,2 \mathrm{Aa}$ & $497,6 \mathrm{Aa}$ & 8,14 \\
\hline
\end{tabular}

Médias seguidas de mesma letra maiúscula na coluna e de mesma letra minúscula na linha não diferem entre si, a 5\% de probabilidade, pelo teste de Tukey.

De acordo com os resultados apresentados na Tabela 4, observa-se que o valor de deslizamento das rodas motrizes dentro de cada condição de subsolagem foi estatisticamente o mesmo; todavia, ao confrontar o deslizamento de cada sistema de preparo, numa condição versus a outra, verificou-se que, apesar da menor aderência dos pneus, a subsolagem depois do preparo foi mais vantajosa, pois resultou em deslizamento médio $15,2 \%$ menor do que na condição de subsolagem antes do preparo. O menor deslizamento das rodas motrizes proporciona menor compactação do solo, menor desgaste dos pneus e dos mecanismos de transmissão, como também reduz os gastos adicionais de combustíveis. A redução do deslizamento traduziu-se em aumento significativo na velocidade média operacional, com conseqüente aumento no rendimento operacional.

Os resultados da velocidade média operacional tiveram desempenho semelhante aos de deslizamento, uma vez que se correlacionam negativamente (Tabela 5). A comparação dos resultados numa condição versus a outra, dentro de cada sistema de preparo, evidencia a superioridade da subsolagem realizada depois do preparo periódico (DP), pois elevou a velocidade média operacional de 9,$1 ; 12,3$ e 12,3\% nos sistemas de preparo D, Dn e Gn, respectivamente. Devido à menor camada de solo firme a ser rompida pelas hastes, a média das velocidades na condição DP foi $8,2 \%$ maior do que na condição de subsolagem antes do preparo, apesar da menor aderência e da maior resistência ao rolamento oferecidas pela superfície de solo mobilizada. 
TABELA 4. Deslizamento dos rodados motrizes (\%), em função das condições de subsolagem e dos sistemas de preparo do solo. Slip wheel (\%), depending on the conditions of subsoiling and systems of soil preparation.

\begin{tabular}{lccc}
\hline \multicolumn{3}{c}{ Deslizamento (\%) } & \multirow{2}{*}{ Diferença (\%) } \\
\cline { 2 - 3 } Sistemas de Preparo & \multicolumn{2}{c}{ Condições de Subsolagem } & \\
\cline { 2 - 3 } $\mathrm{D}$ & $28,36 \mathrm{Ab}$ & $\mathrm{DP}$ & $-23,8$ \\
$\mathrm{Dn}$ & $28,77 \mathrm{Ab}$ & $20,34 \mathrm{Aa}$ & $-29,3$ \\
$\mathrm{G}$ & $25,82 \mathrm{Aa}$ & $25,09 \mathrm{Aa}$ & $-2,8$ \\
$\mathrm{Gn}$ & $29,10 \mathrm{Ab}$ & $21,73 \mathrm{Aa}$ & $-25,3$ \\
$\mathrm{E}$ & $26,43 \mathrm{Ab}$ & $22,13 \mathrm{Aa}$ & $-16,3$ \\
\hline Média & $27,70 \mathrm{~b}$ & $22,18 \mathrm{a}$ & $-19,9$ \\
\hline \multicolumn{2}{l}{ Médias seguidas de mesma letra maiúscula na coluna e de mesma letra minúscula na linha não diferem entre si, a 5\% de } \\
probabilidade, pelo teste de Tukey.
\end{tabular}

TABELA 5. Velocidade média operacional, em função das condições de subsolagem e dos sistemas de preparo. Average operational speed $\left(\mathrm{km} \mathrm{h}^{-1}\right)$, as related to the subsoiling conditions and tillage systems.

\begin{tabular}{lccc}
\hline & \multicolumn{2}{c}{ Velocidade Média Operacional $\left(\mathrm{km} \mathrm{h}^{-1}\right)$} & \\
\cline { 2 - 3 } Sistema de Preparo & \multicolumn{2}{c}{ Condições de Subsolagem } & \multirow{2}{*}{ Diferença (\%) } \\
\cline { 2 - 3 } $\mathrm{D}$ & $\mathrm{AP}$ & $\mathrm{DP}$ & 9,1 \\
$\mathrm{Dn}$ & $2,10 \mathrm{Ab}$ & $2,31 \mathrm{Aa}$ & 12,3 \\
$\mathrm{G}$ & $2,06 \mathrm{Ab}$ & $2,35 \mathrm{Aa}$ & 2,3 \\
$\mathrm{Gn}$ & $2,17 \mathrm{Aa}$ & $2,22 \mathrm{Aa}$ & 12,3 \\
$\mathrm{E}$ & $2,10 \mathrm{Ab}$ & $2,39 \mathrm{Aa}$ & 4,9 \\
\hline Média & $2,14 \mathrm{Aa}$ & $2,25 \mathrm{Aa}$ & 8,2 \\
\hline
\end{tabular}

Médias seguidas de mesma letra maiúscula na coluna e de mesma letra minúscula na linha não diferem entre si, a 5\% de probabilidade, pelo teste de Tukey.

\section{CONCLUSÕES}

A realização da subsolagem depois dos sistemas de preparo periódico requereu $15 \%$ menos de potência na barra de tração. A subsolagem depois dos diferentes sistemas de preparo economizou $16,5 \%$ de combustível por área. O deslizamento das rodas motrizes e a velocidade média operacional obtiveram melhor desempenho quando se realizou a subsolagem depois do preparo do solo.

\section{REFERÊNCIAS}

BURT, E.C.; LYNE, P.W.L.; MEIRING, P. Ballast and inflation effects on tire tractive. Transactions of the ASAE, St. Joseph, v.26, n.5, p.1.352-4, 1983.

EL-ARABY, A.; EL-HADDAD, Z.; EL-ANSARY, M. Subsoiling in some heavy olay soils of Egypt. Soil \& Tilage Research, Amsterdam, v.9, n.3, p.207-16, 1987.

EMBRAPA. EMPRESA BRASILEIRA DE PESQUISA AGROPECUÁRIA. Centro Nacional de Pesquisas de Solos. Sistema Brasileiro de Classificação de Solos. Brasília, 1999. 412 p.

FORNSTRON, K.J.; BECKER, C.G. Comparison of energy requeriments and machinery for four sumer fallow methods. Transactions of the ASAE, St. Joseph, v.20, n.4, p.640-2, 1977.

KICHLER, C.M.; FULTON, P.R.J.; RAPER, L.; ZECH, W.C.; MCDONALD, T.P.; BRODBECK, C.J. Spatially monitoring tractor performance to evaluate energy requirements of variable depth 
tillage and implement selection. In: ASABE ANNUAL INTERNATIONAL MEETING, Minneapolis, 2007. Proceedings... St. Joseph: ASABE, 2007. Paper Number: 071028. 2007

LANÇAS, K.P. Subsolador: desempenho em função de formas geométricas de hastes, tipos de ponteiras e velocidade de deslocamento. 1987. 112 f. Dissertação (Mestrado em Energia na Agricultura) - Faculdade de Ciências Agronômicas, Universidade Estadual Paulista, Botucatu, 1987.

LANÇAS, K.P. Subsolador: desempenho em função de formas geométricas de hastes, tipos de ponteiras e número de hastes. 1988. 171 f. Tese (Doutorado em Energia na Agricultura) - Faculdade de Ciências Agronômicas, Universidade Estadual Paulista, Botucatu, 1988.

LYNE, P. W. L.; BURT, E. C.; MEIRING, P. Effect of tire and engine parameters on efficiency. Transactions of the ASAE, St. Joseph, v.27, n.1, p.5-7, 11, 1984.

MIRANDA, N. de O.; OLIVEIRA, M. de; NUNES, R.L. Desempenho operacional de trator com tração dianteira auxiliar na subsolagem de um inceptisol. Revista Brasileira de Engenharia Agrícola e Ambiental, Campina Grande, v.4, n.1, p.97-102, 2000.

RAPER, R.L.; REEVES, D.W.; SHAW, J.N.; VAN SANTEN, E.; MASK, P.L. Site-specific subsoiling benefits for cotton production. St. Joseph: ASABE, 2005. ASAE Paper 051025. 